\title{
Potential roles of reactive oxygen species derived from chemical substances involved in cancer development in the female reproductive system
}

\author{
Soo-Min Kim, Kyung-A Hwang ${ }^{*} \mathcal{E}$ Kyung-Chul Choi * \\ Laboratory of Biochemistry and Immunology, College of Veterinary Medicine, Chungbuk National University, Cheongju 28644, Korea
}

\begin{abstract}
Reactive oxygen species (ROS) are major sources of cellular oxidative stress. Specifically, cancer cells harbor genetic alterations that promote a continuous and elevated production of ROS. While such oxidative stress conditions could be harmful to normal cells, they facilitate cancer cell growth in multiple ways by causing DNA damage and genomic instability, and ultimately by reprogramming cancer cell metabolism. This review provides up to date findings regarding the roles of ROS generation induced by diverse biological molecules and chemicals in representative women's cancer. Specifically, we describe the cellular signaling pathways that regulate direct or indirect interactions between ROS homeostasis and metabolism within female genital cancer cells. [BMB Reports 2018; 51(11): 557-562]
\end{abstract}

\section{INTRODUCTION}

Oxidative stress is caused by an imbalance in reactive oxygen species (ROS). The regulation of ROS homeostasis plays a major role in cellular growth, metabolism, and survival (1). When present at low levels, ROS is an important signaling molecule that can maintain cellular functions such as viability, migration and apoptosis (2). However, the excessive occurrence of ROS causes biological systems to incompletely detoxify the reactive intermediates and to block the normal functions of biomolecules (3). Both ROS and oxidative stress are considered to be involved in aging (4), inflammation (5), and many diseases, including cancer because ROS plays a

*Corresponding authors. Kyung-Chul Choi, Tel: +82-43-261-3664; Fax: +82-43-267-3150; E-mail: kchoi@cbu.ac.kr; Kyung-A Hwang, Tel: +82-43-249-1745; Fax: +82-43-267-3150; E-mail: hka9400@ naver.com

https://doi.org/10.5483/BMBRep.2018.51.11.056

Received 18 March 2018, Revised 10 April 2018, Accepted 18 June 2018

Keywords: Apoptosis, Cancer development, Choriocarcinoma cell, Ovarian and endometrial cancers, Reactive oxygen species significant role in the post-transcriptional adjustment of genes and repercussion effects on cellular development, differentiation, proliferation, apoptosis and the development and progression of cancer $(6,7)$.

Furthermore, excessive ROS may affect women's diseases occurring in reproductive organs (8). Endometriosis has been found to be correlated with proinflammatory mediators and ROS, which can lead to cellular proliferation and activation of ERK1/2 (9). In addition, the toxic effects of ROS have been shown to induce malignancy of ovarian cancer (OC) through reduced expression of antioxidant enzymes (10).

This review describes the adaptive mechanisms that cancer cells in women's reproductive organs take to face oxidative stress conditions. We will discuss the role of ROS induced by diverse biological molecules and chemicals in regulating the related signaling pathways and consequential oxidative stressmediated responses in female reproductive cancers occurring in organs such as the placenta, ovary, and endometrium.

\section{RELATIONSHIP BETWEEN ROS AND CANCER}

Oxidative stress has been reported to affect all phases of the oncogenic process including initiation, promotion, and progression $(11,12)$. Under the impact of ROS in the cancer development stage (Fig. 1), ROS can induce altered expression of several transcription factors associated with regulating pathways such as genetic mutations, proliferation, suppression, differentiation, and senescence $(8,13)$.

A variety of chemical substances and naturally occurring chemicals can mediate carcinogenesis, malignant behavior, and treatment response in cancer development via regulation of ROS imbalance. For instance, several studies have shown that cancer cells induce apoptosis or necrosis of damaged cells to maintain homeostasis of ROS and prevent ROS-induced toxicity. This process is thought to be the initiation of cancer development (13). Moreover, when the oxidative stress state of cells persists from the imbalance of ROS, DNA damage and induction of mutations affect cellular signaling pathways (14, $15)$, leading to the activation of a variety of protein kinases that regulate diverse cellular functions including the cell cycle, survival, migration, angiogenesis, apoptosis and cell death 


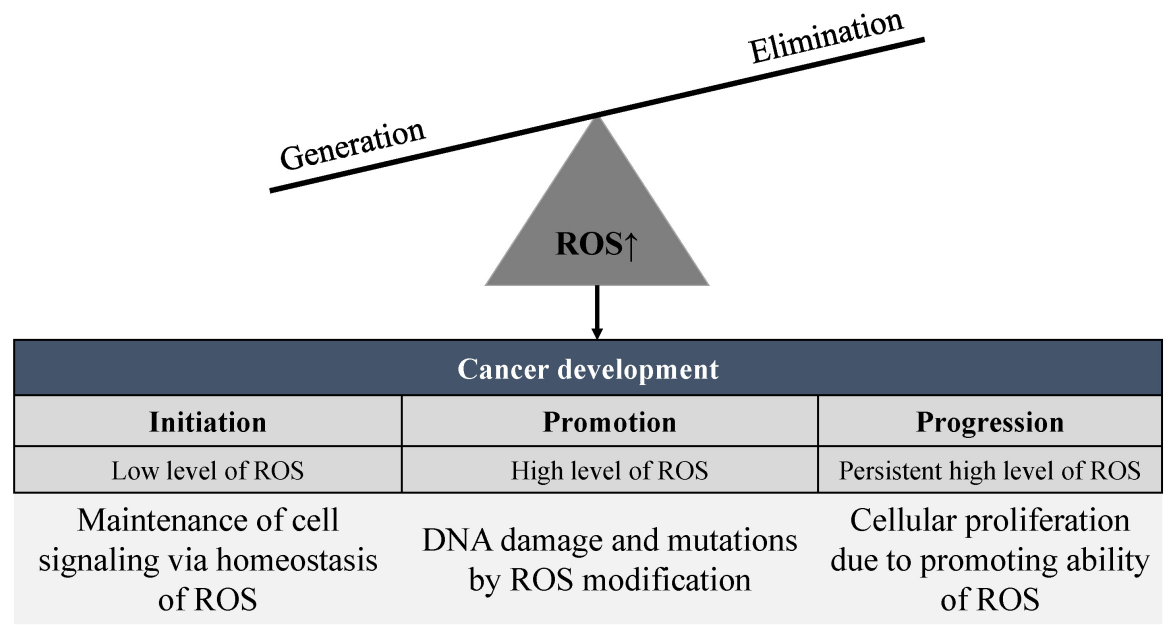

Fig. 1. Relationship between ROS production and cancer cell development. Initiation, the first step among three stages in cancer, maintains the homeostasis of ROS against imbalance of ROS and regulates protein kinases, which have diverse cellular functions. Promotion, the second step, is related to DNA damage and induction of mutations appearing in the cellular signaling pathway. Progression, the third step, appears to activate EMTrelated genes and other intracellular signaling pathway markers. Therefore, the generation of ROS is crucial to the three developmental stages of cancer; initiation, promotion, and progression.
(16). The infinite cell proliferation capacity of cancer cells has an effect on angiogenesis related mechanisms (17), which can induce cancer metastasis by promoting cancer progressionassociated processes such as proliferation, migration, and tube formation (18).

Generally, there are a variety of ways to treat cancer, including surgery, chemotherapy, radiation, immunotherapy, and other targeted therapies. Among them, chemotherapy and radiation therapy lead to the generation of ROS with strong toxicity to cancer cells (19). Specifically, it has been reported that the production of ROS also affects mitogen-activated protein kinases (MAPK) (20) that act as a switch to block or transmit signals because of the phosphorylation of surrounding proteins. Moreover, activation of the MAPK pathway can result in apoptosis via death signals including the c-Jun $\mathrm{N}$-terminal kinase (JNK) pathway and caspase family (21). The cancer cells that continue to proliferate tend to induce DNA damage and to elicit cell cycle arrest $(22,23)$. Therefore, excessive production of ROS can provoke cell cycle arrest, apoptosis, and senescence. Additionally, ROS is known to be correlated with malignant progression of cancer cells by increasing invasion and metastatic potentials via MAPK signals (13). ROS-stimulated growth factors and Ras (renin-angiotensin system) in cancer cells play a role in inducing activation of the MAPK pathway, and the activated Ras-MAPK pathway has been shown to lead to cell proliferation $(24,25)$ and extracellular matrix $(\mathrm{ECM})$ alteration via the upregulation of specific matrix metalloproteinases (MMPs) (26). The upregulated MMP then leads to the invasion via degradation of ECM (27). Successively, the decomposed ECM collapses the layer structures and then enables cancer cells to migrate. Therefore, ROS has been shown to activate the MAPK pathway leading to cancer progression and dissemination (24, 28).

Most of the tumorigenic activities of ROS are associated with regulation of transcription factors such as activator protein 1 (AP-1), nuclear factor- $\kappa \mathrm{B}$ (NF- $\kappa \mathrm{B})$, nuclear factor erythroid 2-related factor 2 (Nrf2), and hypoxia-inducible factor- $1 \alpha(\mathrm{HIF}-1 \alpha)$, as well as intercellular adhesion protein-1 (ICAM-1) and p53 (29). Moreover, diverse in-depth molecular regulatory mechanisms of ROS-induced cellular reactions under pathological conditions, including cancer, have been reported in previous reviews $(13,30)$. In this review, we will focus on cellular modulations induced by chemicals or natural substances-induced formation of ROS in women's cancers.

\section{ROLES OF ROS IN FEMALE REPRODUCTIVE CANCERS}

\section{Choriocarcinoma}

Human placenta related to pregnancy can develop and lead to specialized fetal trophoblasts; therefore, it plays an important role in implantation and development of the maternal-fetal interface $(31,32)$. Choriocarcinoma (CC) is a rare cancer occurring in the trophoblastic cells and cytotrophoblast, which form a chronic membrane of the placenta (33). The form of CC is known to be a malignant trophoblastic tumor that quickly spreads to the organs from the uterus. The metastasis of CC occurs via hematogenous routes to the liver, brain, etc., although the most common site is the lungs (34).

Commonly, chemotherapy has a significant curative influence on CC. However, drugs generally used in cancer therapy have barriers such as drug resistance and side effects. Therefore, patients with refractory gestational CC do not have an optimistic outlook when being treated with chemotherapy (35). Chemotherapy and radiation therapy to remove cancer cells usually increase intracellular ROS and damage many other biomolecules $(36,37)$. Moreover, the cellular ROS concentrations may have been implicated in the selective activation of transcription factors, and either cell death or cell proliferation may result from exposure to oxidative stress (38). Here, we provide representative examples that show cellular adjustment to oxidative stress induced by chemical agents in 
CC.

Huovinen et al. evaluated the effects of diuron as an endocrine disruptor, which produced adverse development and reproductive effects in BeWo cells (a human CC model). Moreover, diuron appeared to produce ROS and to inhibit cell proliferation of BeWo cells because the protein expression of p53 as a biomarker for cell stress and p21 as a cell cycle arrest gene was increased by oxidative stress $(39,40)$. A study by Ham et al. revealed that silibinin, a flavonolignan with anti-cancer effects extracted from seeds of milk thistles, significantly inhibited proliferation and induced apoptosis in both JAR and JEG3 CC cells by increasing ROS production and lipid peroxidation. Moreover, silibinin interrupted mitochondrial function by inducing mitochondrial membrane potential and permeabilization of calcium ion efflux in these cancer models (41).

As another phytochemical, coumestrol was shown to induce cell death by regulating ERK $1 / 2$ MAPK and JNK MAPK signaling pathways through disruption of $\mathrm{Ca}^{2+}$ and ROS homeostasis. Specifically, coumestrol suppressed proliferation and increased apoptosis of JAR and JEG3 cells by inducing the pro-apoptotic proteins, Bax and Bak, via ROS production and lipid peroxidation. Coumestrol also induced depolarization of MMP and increased cytosolic and mitochondrial $\mathrm{Ca}^{2+}$ levels in JAR and JEG3 cells, leading to apoptosis of CC cells through regulation of cell signaling and mitochondrial-mediated functions with a potential to impair progression of the cancer (42). A similar study evaluated the effects of chrysophanol, an anthraquinone compound, on JAR and JEG-3 cells. These results showed that chrysophanol decreased cell viability and induced apoptosis, while increasing oxidative stress in JEG-3 cells by inducing ROS generation followed by mitochondrial dysfunction, including depolarization of the mitochondrial inner membrane potential. In this experiment, the ERK $1 / 2$ and AKT signaling pathways were significantly activated in JEG-3 cells by ROS (42).

In another study, benzo(a)pyrene decreased cell viability and induced cell cycle arrest by increasing the ROS level in CC cells. The increased ROS levels enabled induction of apoptosis and simultaneous activation of endoplasmic reticulum (ER) stress (43). These studies confirm that ROS induced by diverse stimuli plays an important role in the induction of apoptosis in CC cells.

However, another study showed that formaldehyde and benzene increased the proliferation and migration of JEG-3 cells and epithelial mesenchymal transition (EMT) during ROS production (43). In this case, the increased level of ROS promoted the cancer progression of $\mathrm{CC}$, unlike in previous studies. Taken together, these findings suggest that ROS-related mechanisms in CC are associated with cancer progression as well as cell death of CC.

\section{Ovarian cancer}

Ovarian cancer is the fourth most common cause of cancer death in gynecological malignancies (44) and the second most diagnosed cancer among gynecologic malignant tumors (45). Ovarian cancer develops through the formation of a neoplasm in tissues of an ovary, and epithelial ovarian cancer is a major type of cancer $(46,47)$. Most advanced stage cancers originate from epithelial cells, although some originate from serous, mucinous, or endometrioid cells into the surface epithelium of the ovary or fallopian tube (48). The representative metastatic sites of OC are the endometrium, breast, colon, and stomach, and OC migrates through the body's blood stream and lymph fluid (49). Most cases of OC are difficult to treat because they are diagnosed at highly advanced stages $(45,50,51)$. The advanced stage of OC is closely associated with high levels of ROS, which produces a large amount of hydrogen peroxide, whereas oxidative stress is induced in the oxidizing environment of the tumor (52).

Zet et al. previously sought to determine the effects of inhibiting intracellular ROS generation in epithelial ovarian cancer (EOC) cells. As a result, treatment with diphenyleneiodonium (DPI), a ROS inhibitor, significantly induced apoptosis in EOC cells by increasing caspase-3 activity. Moreover, DPI treatment resulted in reduced NADPH oxidase, SOD3 and HIF-1 $\alpha$ levels in EOC cells (10), indicating that lowering oxidative stress, possibly through the inhibition of NADPH oxidase, induces apoptosis in OC cells.

\section{Endometrial cancer}

Endometrial cancer (EC) is a representative malignant gynecologic carcinoma that is the most common cancer in women except for breast cancer (53). The majority of endometrial malignancies (95\%) occur in endometrial glands and are known as endometrial cancers. The remaining 5\% occur in mesenchymal tumors and are known as carcinosarcoma (mixed epithelium/epilepsy tumor) (54).

Because of early symptoms such as abnormal uterine bleeding or pelvic pain, EC is often diagnosed at an early stage. However, this cancer, which is characterized by endometrial and lymphatic invasion, sometimes manifests as biologically aggressive mutations (55). Molecular alterations can induce cellular regulations, which play an important role in the development of EC (53).

Ellipticine, an alkaloid isolated from Apocyanaceae plants, has been shown to induce apoptosis in RL95-2 human EC cells via ROS formation. Ellipticine-induced apoptosis was found to be associated with the arrest of cells in the G2/M phase and accompanied by depolarization of the mitochondrial membrane potential, release of cytochrome $\mathrm{c}$ and apoptosisinducing factor (AIF) from the mitochondrial membrane and activation of caspase. In this case, ROS accumulation was shown to activate the ERK and JNK pathway and finally to release AIF in the RL95-2 cells (56).

(-)-Epigallocatechin-3-gallate (EGCG), the major polyphenol in green tea, has been shown to have anti-proliferative potential on human Ishikawa endometrial cancer cells. In this 
Table 1. Outcomes of various biomolecular pathways resulting from the production of ROS in gynecological cancers

\begin{tabular}{|c|c|c|}
\hline Cancer & Biomolecular process related to ROS & Reference \\
\hline Choriocarcinoma cancer & $\begin{array}{l}\text { - Inhibition of cell proliferation via cell cycle arrest and mitochondrial dysfunction } \\
\text { - EMT through controlling the cell cycle and migration ability }\end{array}$ & $\begin{array}{l}(39-41) \\
(43)\end{array}$ \\
\hline Ovarian cancer & - Apoptosis through inhibition of NADPH oxidase & $(10)$ \\
\hline Endometrial cancer & $\begin{array}{l}\text { - Apoptosis via activation of the ERK and JNK pathways } \\
\text { - Inhibition of cell proliferation via activation of p38 } \\
\text { - Metastasis or invasion via EMT-related transcription factors }\end{array}$ & $\begin{array}{l}(56,58) \\
(57) \\
(59)\end{array}$ \\
\hline
\end{tabular}

process, EGCG inhibited ERK and its downstream transcription factors fos and jun through marked enhancement of ROS and activation of p38 in Ishikawa cells. These results suggest that inhibition of ERK activation and induction of apoptosis through ROS generation and p38 activation may affect the pathway to inhibit proliferation (57). Similar effects have been reported in the study using HEC-1A EC cells, in which ROS induced apoptosis and inhibition of cell growth (58).

The analysis of proteins under the Ets variant 5 (ETV5)related proteome approach in the HEC-1A cell line reinforced the role of transcription factor in the regulation of metastatic and invasive tumor behavior in EC and showed a regulatory response to oxidative stress associated with endometrial invasion enhancement (59). Based on this research, it can be assumed that ROS production affects pathways involved in metastasis or invasion in EC cells.

\section{CONCLUSION}

Oxidative stress is known to be related to the pathogenesis of various malignant cancers, and this review specifically discussed the effects of biomolecules associated with ROS production in representative female cancers. We focused on ROS effects on apoptosis and cell proliferation in CC, OC, and EC.

First, we reviewed that the occurrence of ROS is crucial to the development of cancer (initiation, promotion, and progression) as shown in Fig. 1. Initiation, the first step, was shown to maintain the homeostasis of ROS against imbalance of ROS and to regulate protein kinases, which have diverse cellular functions. Promotion, the second step, is related to DNA damage and induction of mutations appearing in cellular signaling pathway by ROS. Progression, the third step, appears to activate EMT-related genes and other intracellular signaling pathway markers by ROS.

According to a great deal of research data, the formation of ROS indicates various responses in gynecological cancers through activation of the signal transduction pathway as shown in Table 1. The current pathological evidence suggests that there is a correlation between the production of ROS and the progression of female cancers.

Currently, there is a need for further investigation to understand the biological and pathological features of ROS in female cancers because ROS-related effects are not uniform, and are instead associated with cancer progression and cancer cell death depending on the cancer types and ROS formation conditions. Therefore, understanding how the imbalance of ROS regulation affects the developmental tendency of cancer can help develop strategies that interfere with cancer development. Further information regarding this content will provide useful predictive factors and potential therapeutic targets for female cancer patients undergoing chemotherapy related to ROS intervention.

\section{ACKNOWLEDGEMENTS}

This work was supported by a National Research Foundation of Korea (NRF) grant funded by the Ministry of Education, Science and Technology (MEST) of the Republic of Korea (2017R1D1A1A09000663). In addition, this work was also supported by a grant (no.317021-03-1-CG000) from the Korea Institute of Planning and Evaluation for technology in Food, Agriculture and Forestry, Republic of Korea.

\section{CONFLICTS OF INTEREST}

The authors have no conflicting interests.

\section{REFERENCES}

1. Kim J, Kim J and Bae JS (2016) ROS homeostasis and metabolism: a critical liaison for cancer therapy. Exp Mol Med 48, e269

2. Chrissobolis $S$ and Faraci FM (2008) The role of oxidative stress and NADPH oxidase in cerebrovascular disease. Trends Mol Med 14, 495-502

3. Gius D and Spitz DR (2006) Redox signaling in cancer biology. Antioxid Redox Signal 8, 1249-1252

4. Galaris D, Mantzaris M and Amorgianiotis C (2008) Oxidative stress and aging: the potential role of iron. Hormones (Athens) 7, 114-122

5. Van Wagoner DR (2008) Oxidative stress and inflammation in atrial fibrillation: role in pathogenesis and potential as a therapeutic target. J Cardiovasc Pharmacol 52, 306-313

6. Rojas A, Silva R, Figueroa $\mathrm{H}$ and Morales MA (2008) Oxidative stress in tumor microenvironment--lts role in 
angiogenesis. Zhongguo Fei Ai Za Zhi 11, 297-305

7. Giannoni E, Parri M and Chiarugi P (2012) EMT and oxidative stress: a bidirectional interplay affecting tumor malignancy. Antioxid Redox Signal 16, 1248-1263

8. Marengo B, Nitti M, Furfaro AL et al (2016) Redox Homeostasis and Cellular Antioxidant Systems: Crucial Players in Cancer Growth and Therapy. Oxid Med Cell Longev 2016, 6235641

9. Ngo C, Chereau C, Nicco C, Weill B, Chapron C and Batteux F (2009) Reactive oxygen species controls endometriosis progression. Am J Pathol 175, 225-234

10. Jiang Z, Fletcher NM, Ali-Fehmi R et al (2011) Modulation of redox signaling promotes apoptosis in epithelial ovarian cancer cells. Gynecol Oncol 122, 418-423

11. Bhat AV, Hora S, Pal A, Jha S and Taneja R (2017) Stressing the (epi)genome: dealing with ROS in cancer. Antioxid Redox Signal [Epub ahead of print]

12. Strzelczyk JK and Wiczkowski A (2012) Oxidative damage and carcinogenesis. Contemp Oncol (Pozn) 16, 230-233

13. Panieri E and Santoro MM (2016) ROS homeostasis and metabolism: a dangerous liason in cancer cells. Cell Death Dis 7, e2253

14. Moloney JN and Cotter TG (2018) ROS signalling in the biology of cancer. Semin Cell Dev Biol 80, 50-64

15. Sieber OM, Heinimann K and Tomlinson IP (2003) Genomic instability-the engine of tumorigenesis? Nat Rev Cancer 3, 701-708

16. Gao X and Schottker B (2017) Reduction-oxidation pathways involved in cancer development: a systematic review of literature reviews. Oncotarget 8, 51888-51906

17. Morry J, Ngamcherdtrakul W and Yantasee W (2017) Oxidative stress in cancer and fibrosis: Opportunity for therapeutic intervention with antioxidant compounds, enzymes, and nanoparticles. Redox Biol 11, 240-253

18. Beckman RA and Loeb LA (2017) Evolutionary dynamics and significance of multiple subclonal mutations in cancer. DNA Repair (Amst) 56, 7-15

19. Chan L, He L, Zhou B et al (2017) Cancer-targeted Selenium Nanoparticles Sensitize Cancer Cells to Continuous gamma Radiation to Achieve Synergetic Chemo-Radiotherapy. Chem Asian J 12, 3053-3060

20. He H, Chang R, Zhang T, Yang C and Kong Z (2017) ATM mediates DAB2IP-deficient bladder cancer cell resistance to ionizing radiation through the p38MAPK and NF-kappaB signaling pathway. Mol Med Rep 16, $1216-1222$

21. Xu Z, Zhang F, Bai C et al (2017) Sophoridine induces apoptosis and S phase arrest via ROS-dependent JNK and ERK activation in human pancreatic cancer cells. J Exp Clin Cancer Res 36, 124

22. Preya UH, Lee KT, Kim NJ, Lee JY, Jang DS and Choi JH (2017) The natural terthiophene alpha-terthienylmethanol induces $\mathrm{S}$ phase cell cycle arrest of human ovarian cancer cells via the generation of ROS stress. Chem Biol Interact $272,72-79$

23. Khan F, Khan I, Farooqui A and Ansari IA (2017) Carvacrol Induces Reactive Oxygen Species (ROS)-mediated Apoptosis Along with Cell Cycle Arrest at G0/G1 in Human Prostate Cancer Cells. Nutr Cancer 69, 1075-1087
24. McCubrey JA, Steelman LS, Chappell WH et al (2007) Roles of the Raf/MEK/ERK pathway in cell growth, malignant transformation and drug resistance. Biochim Biophys Acta 1773, 1263-1284

25. Liu CT and Liu MY (2017) Daily sesame oil supplementation attenuates local renin-angiotensin system via inhibiting MAPK activation and oxidative stress in cardiac hypertrophy. J Nutr Biochem 42, 108-116

26. Huang H, Du W and Brekken RA (2017) Extracellular Matrix Induction of Intracellular Reactive Oxygen Species. Antioxid Redox Signal 27, 774-784

27. $\mathrm{Ha} \mathrm{H}$ and Lee HB (2003) Reactive oxygen species and matrix remodeling in diabetic kidney. J Am Soc Nephrol 14, S246-249

28. Seth D and Rudolph J (2006) Redox regulation of MAP kinase phosphatase 3. Biochemistry 45, 8476-8487

29. Klaunig JE, Kamendulis LM and Hocevar BA (2010) Oxidative stress and oxidative damage in carcinogenesis. Toxicol Pathol 38, 96-109

30. Galadari S, Rahman A, Pallichankandy S and Thayyullathil F (2017) Reactive oxygen species and cancer paradox: To promote or to suppress? Free Radic Biol Med 104, 144-164

31. Red-Horse K, Zhou Y, Genbacev O et al (2004) Trophoblast differentiation during embryo implantation and formation of the maternal-fetal interface. J Clin Invest $114,744-754$

32. Hod T, Cerdeira AS and Karumanchi SA (2015) Molecular Mechanisms of Preeclampsia. Cold Spring Harb Perspect Med 5, a023473

33. Kumar V, Abbas AK and Aster JC (2015) Robbins and Cotran pathologic basis of disease, Ninth edition. ed, Elsevier/Saunders, Philadelphia, PA

34. Park SH, Park A, Kim JY, Kwon JH and Koh SB (2009) A case of non-gestational choriocarcinoma arising in the ovary of a postmenopausal woman. J Gynecol Oncol 20, 192-194

35. Lurain JR and Nejad B (2005) Secondary chemotherapy for high-risk gestational trophoblastic neoplasia. Gynecol Oncol 97, 618-623

36. Renschler MF (2004) The emerging role of reactive oxygen species in cancer therapy. Eur J Cancer 40, 1934-1940

37. Yedjou CG and Tchounwou PB (2012) In vitro assessment of oxidative stress and apoptotic mechanisms of garlic extract in the treatment of acute promyelocytic leukemia. J Cancer Sci Ther 2012, 6

38. Vallejo MJ, Salazar L and Grijalva M (2017) Oxidative Stress Modulation and ROS-Mediated Toxicity in Cancer: A Review on In Vitro Models for Plant-Derived Compounds. Oxid Med Cell Longev 2017, 4586068

39. Huovinen M, Loikkanen J, Naarala J and Vahakangas $K$ (2015) Toxicity of diuron in human cancer cells. Toxicol In Vitro 29, 1577-1586

40. Heazell AE, Taylor NN, Greenwood SL, Baker PN and Crocker IP (2009) Does altered oxygenation or reactive oxygen species alter cell turnover of BeWo choriocarcinoma cells? Reprod Biomed Online 18, 111-119

41. Ham J, Lim W, Bazer FW and Song G (2018) Silibinin 
stimluates apoptosis by inducing generation of ROS and ER stress in human choriocarcinoma cells. J Cell Physiol 233, 1638-1649

42. Lim W, Yang C, Jeong M, Bazer FW and Song G (2017) Coumestrol induces mitochondrial dysfunction by stimulating ROS production and calcium ion influx into mitochondria in human placental choriocarcinoma cells. Mol Hum Reprod 23, 786-802

43. Kim SM, Lee HM, Hwang KA and Choi KC (2017) Benzo(a)pyrene induced cell cycle arrest and apoptosis in human choriocarcinoma cancer cells through reactive oxygen species-induced endoplasmic reticulum-stress pathway. Food Chem Toxicol 107, 339-348

44. Jeon SY, Hwang KA and Choi KC (2016) Effect of steroid hormones, estrogen and progesterone, on epithelial mesenchymal transition in ovarian cancer development. J Steroid Biochem Mol Biol 158, 1-8

45. Rojas V, Hirshfield KM, Ganesan S and RodriguezRodriguez L (2016) Molecular Characterization of Epithelial Ovarian Cancer: Implications for Diagnosis and Treatment. Int J Mol Sci 17, E2113

46. Naora $\mathrm{H}$ and Montell DJ (2005) Ovarian cancer metastasis: integrating insights from disparate model organisms. Nat Rev Cancer 5, 355-366

47. Auersperg N, Wong AS, Choi KC, Kang SK and Leung PC (2001) Ovarian surface epithelium: biology, endocrinology, and pathology. Endocr Rev 22, 255-288

48. Bell DA (2005) Origins and molecular pathology of ovarian cancer. Mod Pathol 18 Suppl 2, S19-32

49. Fidler IJ (2002) The organ microenvironment and cancer metastasis. Differentiation 70, 498-505

50. Holschneider $\mathrm{CH}$ and Berek JS (2000) Ovarian cancer: epidemiology, biology, and prognostic factors. Semin Surg Oncol 19, 3-10

51. McCluggage WG (2011) Morphological subtypes of ovarian carcinoma: a review with emphasis on new developments and pathogenesis. Pathology 43, 420-432

52. Glorieux C, Sandoval JM, Fattaccioli A et al (2016) Chromatin remodeling regulates catalase expression during cancer cells adaptation to chronic oxidative stress. Free Radic Biol Med 99, 436-450

53. Tsikouras P, Bouchlariotou S, Vrachnis $N$ et al (2013) Endometrial cancer: molecular and therapeutic aspects. Eur J Obstet Gynecol Reprod Biol 169, 1-9

54. Llaurado M, Ruiz A, Majem B et al (2012) Molecular bases of endometrial cancer: new roles for new actors in the diagnosis and the therapy of the disease. Mol Cell Endocrinol 358, 244-255

55. Ali AT (2013) Risk factors for endometrial cancer. Ceska Gynekol 78, 448-459

56. Kim JY, Lee SG, Chung JY et al (2011) Ellipticine induces apoptosis in human endometrial cancer cells: the potential involvement of reactive oxygen species and mitogen-activated protein kinases. Toxicology 289, 91-102

57. Manohar M, Fatima I, Saxena R, Chandra V, Sankhwar PL and Dwivedi A (2013) (-)-Epigallocatechin-3-gallate induces apoptosis in human endometrial adenocarcinoma cells via ROS generation and p38 MAP kinase activation. J Nutr Biochem 24, 940-947

58. Yoshioka T, Yogosawa S, Yamada T, Kitawaki J and Sakai T (2013) Combination of a novel HDAC inhibitor OBP-801/YM753 and a PI3K inhibitor LY294002 synergistically induces apoptosis in human endometrial carcinoma cells due to increase of Bim with accumulation of ROS. Gynecol Oncol 129, 425-432

59. Monge M, Colas E, Doll A et al (2009) Proteomic approach to ETV5 during endometrial carcinoma invasion reveals a link to oxidative stress. Carcinogenesis 30, 1288-1297 